\title{
Development of a novel phantom using polyethylene glycol for the visualization of restricted diffusion in diffusion kurtosis imaging and apparent diffusion coefficient subtraction method
}

\author{
ABDULLAH KHASAWNEH $^{1}$, MASAHIRO KURODA ${ }^{2}$, YUUKI YOSHIMURA ${ }^{2,3}$, IRFAN SUGIANTO ${ }^{1,4}$, \\ BABATUNDE O. BAMGBOSE ${ }^{1}$, KENTARO HAMADA ${ }^{2}$, MAJD BARHAM ${ }^{1}$, NOUHA TEKIKI ${ }^{1}$, \\ KOHEI KONISHI ${ }^{2}$, KOHEI SUGIMOTO ${ }^{2}$, HINATA ISHIZAKA ${ }^{2}$, AKIRA KUROZUMI ${ }^{5}$, \\ TOSHI MATSUSHITA ${ }^{5}$, SEIICHIRO OHNO ${ }^{5}$, SUSUMU KANAZAWA ${ }^{6}$ and JUNICHI ASAUMI ${ }^{1}$ \\ ${ }^{1}$ Department of Oral and Maxillofacial Radiology, Okayama University Graduate School of Medicine, \\ Dentistry and Pharmaceutical Sciences; ${ }^{2}$ Radiological Technology, Graduate School of Health Sciences, \\ Okayama University, Okayama 700-8558; ${ }^{3}$ Department of Radiology Diagnosis, Okayama Saiseikai General Hospital, \\ Okayama 700-8511, Japan; ${ }^{4}$ Department of Oral Radiology, Faculty of Dentistry, Hasanuddin University, \\ Makassar, Sulawesi Selatan 90245, Indonesia; ${ }^{5}$ Central Division of Radiology, Okayama University Hospital; \\ ${ }^{6}$ Department of Radiology, Okayama University Graduate School of Medicine, \\ Dentistry and Pharmaceutical Sciences, Okayama 700-8558, Japan
}

Received December 19, 2019; Accepted September 3, 2020

DOI: 10.3892/br.2020.1359

\begin{abstract}
The present study aimed to investigate whether polyethylene glycol (PEG) phantoms have the potential to be used as standard phantoms for magnetic resonance imaging (MRI) in order to visualize restricted diffusion in diffusion kurtosis imaging (DKI), the ADC subtraction method (ASM) and the apparent diffusion coefficient (ADC). Diffusion-weighted images of 0-120 mM PEG phantoms were captured to create ADC, DKI and ASM images with post-processing. ASM is a recently developed method for restricted diffusion imaging using the readout segmentation of long variable echo-train sequences. As the PEG concentration increases, the ADC value decreases. Conversely, an increase in DKI and ASM values is associated with increasing PEG concentration. Formulae were constructed to represent the association between PEG concentrations and ADC, DKI and ASM values. These formulae can be used to determine the required PEG concentrations to mimic arbitrary ADC, DKI and ASM values of certain diseases, including tumors and infarctions. Validation experiments were conducted using bio-phantoms and clarified that the PEG phantoms cover the range of $\mathrm{ADC}$ and DKI values reported in previous clinical
\end{abstract}

Correspondence to: Professor Masahiro Kuroda, Radiological Technology, Graduate School of Health Sciences, Okayama University, 2-5-1 Shikata-cho, Okayama 700-8558, Japan

E-mail: kurodamd@cc.okayama-u.ac.jp

Key words: magnetic resonance imaging, phantom, restricted diffusion, diffusion kurtosis imaging, apparent diffusion coefficient, apparent diffusion coefficient subtraction method, bio-phantom research using 3T MRI. PEG phantoms may be useful for future MRI research involving restricted diffusion.

\section{Introduction}

Magnetic resonance imaging (MRI) is a widely adopted method used to conduct clinical tumor examination. Specifically, diffusion MRI is utilized for the early diagnosis of tumors and brain infarctions (1-3). The aim of the current study was to evaluate the potential of polyethylene glycol (PEG) phantoms for use as a standard phantom for restricted diffusion in MRI.

In a previous study, Matsuya et al (4) revealed that PEG phantoms may be used as the standard phantom for apparent diffusion coefficient (ADC). ADC, which indicates the average area that a water molecule moves per second, is calculated using diffusion-weighted MR images. In the body, there are two types of water-molecule diffusion (5). The first is free diffusion, which is defined as water-molecule movement in free space without the restriction of barriers. Free diffusion in solution is affected by temperature, viscosity and various other factors. The second type is restricted diffusion, which is defined as water-molecule movement that is impeded by membrane structures of cells in the body (6). ADC accounts for both free and restricted diffusion in MR images.

In recent years, new MR imaging techniques that represent restricted diffusion have been developed. One example of this is diffusion kurtosis imaging (DKI) (7) which has been developed and has been revealed to be as useful as ADC maps in certain clinical trials. A new technique for imaging restricted diffusion has recently been proposed; the ADC subtraction method (ASM) (8).

The current study aimed to evaluate the usability of polyethylene glycol (PEG) phantoms as standard phantoms for 
restricted diffusion in MRI. It was revealed that PEG phantoms may have the potential to be used as the standard phantoms, not only for ADC, but also for restricted diffusion in DKI and ASM. The current study details these phantoms and provides formulae for calculating PEG concentrations that enable us to create phantoms with arbitrary ADC, DKI and ASM values mimicking various tissues and tumors.

\section{Materials and methods}

Polyethylene glycol (PEG) phantom. The phantom (4) comprised: i) PEG (P3640-500G; Sigma-Aldrich; Merck $\mathrm{KGaA}$ ) as a diffusion modifier; ii) $\mathrm{NaN}_{3}$ (Katayama Chemical Industry Co., Ltd.) as an antiseptic; and iii) distilled water.

The phantom solution was heated and diluted using distilled water to achieve concentrations of 20, 40, 60, 80, 100 and $120 \mathrm{mM}$ with $0.03 \% \mathrm{w} / \mathrm{w} \mathrm{NaN}_{3}$. The solution was transferred to microcuvettes (Halbmikro $1.5 \mathrm{ml}$; Greiner Labortechnik Manufacturing Ltd.) and used as the PEG phantoms. Subsequently, the cuvettes were installed in a phantom container (length, $12.5 \mathrm{~cm}$; width, $10.5 \mathrm{~cm}$; and height $9.5 \mathrm{~cm})$ and were immersed in physiological saline $(0.9 \% \mathrm{NaCl})$.

Bio-phantom. A bio-phantom (9) was prepared using Jurkat cells (Bio Resource Center) to ensure that the range of ADC, DKI and ASM values fall within the range of those of the PEG phantoms. Cells were cultured in $10 \%$ fetal bovine serum (Filtron Pty Ltd.), 1\% penicillin-streptomycin-neomycin (Gibco; Thermo Fisher Scientific, Inc.) and RPMI-1640 medium (pH 7.4; Gibco; Thermo Fisher Scientific, Inc.). The incubation was carried out at $37^{\circ} \mathrm{C}$ with $5 \% \mathrm{CO}_{2}$. The number of cells with a diameter $>8 \mu \mathrm{m}$ was counted using an electric cell counter (Beckman Coulter, Inc.) prior to bio-phantom preparation, as the diameter of the majority of Jurkat cells is $>8 \mu \mathrm{m}$ (mean, $9.6 \mu \mathrm{m}$ ). After measuring the cell number, the cell solution was condensed to $\sim 0.89 \mathrm{ml}$, transferred to a micro-cuvette (Halbmikro $1.5 \mathrm{ml}$; Greiner Labortechnik Manufacturing Ltd.), and centrifuged at $161 \mathrm{x} \mathrm{g}$ for $5 \mathrm{~min}$. Thereafter, the supernatant was removed and the resulting bio-phantom was composed of pellet-like cells (density, $\sim 1-8 \times 10^{8}$ cells $/ \mathrm{ml}$ ).

MRI device, image analysis software and statistical analysis software. A 3.0T MRI device (MAGNETOM Prisma VE11C; Siemens AG) was used which had a 20-channel head/neck coil. ImageJ 1.52a (National Institute of Health) was used for image analysis. Diffusional Kurtosis Estimator (DKE) version 2.6 was used for DKI image analysis.

Phantom-heating device. The phantom's temperature was adjusted to a temperature close to that of the human body $\left(\sim 37^{\circ}\right)$ by installing the phantom container in a heating device (constructed in-house) using ethylene-vinyl acetate copolymer. This heating device was connected to a circulating thermostatic chamber (Therm-Mate BF-41; Yamato Scientific Co. Ltd.).

Temperature measurement during MRI. An optic fiber thermometer (FLUOROPTIC ${ }^{\mathrm{TM}}$ m3300; Luxtron Co.) was installed in the microcuvette in the phantom container and was utilized to monitor the phantom's temperature in real time.
Imaging conditions. Table I lists the imaging conditions for DKI and ASM. In DKI, single shot-echo planar imaging (SS-EPI) was used in three sequences; DKI-1, -2 and -3.

In ASM, two types of readout segmentation of long variable echo-train (RESOLVE) sequences were used; RESOLVE-basic and -modify. By changing the number of b-values, two types of DWI were obtained for ASM. For RESOLVE-basic sequences the b-values were set to different points; 0,500 and $1,000 \mathrm{sec} / \mathrm{mm}^{2}$, and for RESOLVE-modify the b-values were set to 4 points: 0,500 , 1,000 and $10,000 \mathrm{sec} / \mathrm{mm}^{2}$ (Table I). As the number of $\mathrm{b}$-values varied, the $\delta$ (motion probing gradient (MPG) pulse duration) and $\Delta$ (MPG pulse spacing) of both sequences changed. In the equation used to calculate b-values (equation 1 ), ' $\Delta-\delta / 3$ ' is termed the effective diffusion time and represents the time during which diffusion phenomena are observed.

$$
\text { Equation 1: } b=\gamma^{2} G^{2} \delta^{2} x(\Delta-\delta / 3)
$$

In the above equation, $\gamma$ represents the gyromagnetic ratio of protons and $\mathrm{G}$ is the gradient magnetic field strength. The $10,000 \mathrm{sec} / \mathrm{mm}^{2} \mathrm{~b}$-value for RESOLVE-modify was used to lengthen the effective diffusion time. The effective diffusion times of RESOLVE-basic and RESOLVE-modify were set to 39.3 and $46.0 \mathrm{msec}$, respectively. Imaging of the PEG phantom was performed 60 times for ADC and ASM and 45 times for DKI.

Image processing of DKI. The DKI analysis software DKE (version 2.6) is available on the website of the Medical University of South Carolina (https://medicine.musc.edu/departments/centers/cbi/dki/dki-data-processing). The DWIs obtained by the imaging of DKI-1, -2 and -3 were processed using DKE to produce a mean kurtosis (MK) image (equation 2), which is a mean value of the spatial direction. By interpolated processing, the voxel size of the MK image becomes 1.0x1.0x1.0 mm.

\section{Equation 2: $\mathrm{S}=\mathrm{S}_{0} \times \exp \left(-\mathrm{b} \times \mathrm{ADC}+\mathrm{b}^{2} \times \mathrm{ADC}^{2} \times \mathrm{MK} / 6\right)$}

In equation $2, \mathrm{~S}$ represents signal intensity and $\mathrm{S}_{0}$ is the signal intensity when the $b$-value $=0 \mathrm{sec} / \mathrm{mm}^{2}$. The $b$-values used are exhibited in Table I.

Image processing of ASM. The $\mathrm{ADC}$ values $\left(\mathrm{ADC}_{\mathrm{b}}\right.$ and $\mathrm{ADC}_{\mathrm{m}}$ ) were calculated for RESOLVE-basic using the b-values: 0, 500 and $1,000 \mathrm{sec} / \mathrm{mm}^{2}$ and for RESOLVE-modify using the above $3 \mathrm{~b}$-values from 0 to $1,000 \mathrm{sec} / \mathrm{mm}^{2}$ only. The $10,000 \mathrm{sec} / \mathrm{mm}^{2} \mathrm{~b}$-value for RESOLVE-modify was not used to calculate the $\mathrm{ADC}_{\mathrm{m}}$ value. The formula used to calculate ASM is as follows:

\section{Equation 3: $\mathrm{ASM}=\mid \mathrm{ADC}_{\mathrm{b}}-\mathrm{ADC}_{\mathrm{m}} \mathrm{l} /\left(\mathrm{ADC}_{\mathrm{b}}\right)^{3}$}

Image evaluation. For MK images, MK values were determined from 1 region of interest (ROI) of $2 \times 2$ pixels in the PEG phantom and from ROIs of the same size in $\leq 6$ areas per image of the physiological saline portion inside the phantom container.

Regarding $\mathrm{ADC}_{\mathrm{b}}$ and $\mathrm{ASM}$, the signal intensity was determined from one ROI of $6 x 6$ pixels selected in the PEG phantom, and from ROIs of the same size in $\leq 6$ areas for each 
Table I. Imaging conditions of DKI and ASM.

\begin{tabular}{|c|c|c|c|c|c|}
\hline \multirow[b]{2}{*}{ Parameters } & \multicolumn{3}{|c|}{ DKI } & \multicolumn{2}{|c|}{ ASM } \\
\hline & DKI-1 & DKI-2 & DKI-3 & RESOLVE-basic & RESOLVE-modify \\
\hline Diffusion time (msec) & 28.9 & 28.9 & - & 39.3 & 46.0 \\
\hline$\delta(\mathrm{msec})$ & 13.8 & 13.8 & - & 5.6 & 15.6 \\
\hline$\Delta(\mathrm{msec})$ & 33.5 & 33.5 & - & 41.2 & 51.2 \\
\hline b-value $\left(\mathrm{sec} / \mathrm{mm}^{2}\right)$ & $0,500,1,000$ & $0,500,1,000$ & 0 & $0,500,1,000$ & $0,500,1,000,10,000$ \\
\hline Imaging time (min:sec) & $6: 24$ & $6: 24$ & $1: 12$ & $13: 28$ & $19: 06$ \\
\hline $\mathrm{TE}(\mathrm{msec})$ & 75 & 75 & 75 & 86 & 106 \\
\hline TR (msec) & 6,000 & 6,000 & 6,000 & 8,000 & 8,000 \\
\hline $\mathrm{ES}(\mathrm{msec})$ & 0.93 & 0.93 & 0.93 & 0.56 & 0.56 \\
\hline FOV (mm) & 120 & 120 & 120 & 120 & 120 \\
\hline Matrix & $82 \times 82$ & $82 \times 82$ & $82 \times 82$ & $224 \times 224$ & $224 \times 224$ \\
\hline BW (Hz/pixel) & 1,220 & 1,220 & 1,220 & 399 & 399 \\
\hline Averages & 1 & 1 & 9 & 2 & 2 \\
\hline Segments & 1 & 1 & 1 & 7 & 7 \\
\hline Slice thickness (mm) & 5 & 5 & 5 & 5 & 5 \\
\hline Slice number & 5 & 5 & 5 & 1 & 1 \\
\hline Phase direction & AP & AP & AP & AP & $\mathrm{AP}$ \\
\hline
\end{tabular}

DKI, diffusion kurtosis imaging; ASM, apparent diffusion coefficient subtraction method; RESOLVE, readout segmentation of long variable echo-trains; $\delta$, motion probing gradient (MPG) pulse duration; $\Delta$, MPG pulse spacing; TE, echo time; TR, repetition time; ES, echo space; FOV, field of view; BW, band width; AP, antero-posterior.

image of the physiological saline portion inside the phantom container. For DWI, signal intensity was determined from ROIs of $6 \times 6$ pixels in the images of RESOLVE-basic and RESOLVE-modify of each b-value. Each signal intensity value was logarithmically transformed. Subsequently, the ADC value for each ROI was calculated using the inverse of the slope and the ASM value was calculated using equation 3.

The total ROIs used for the calculation of the $\mathrm{ADC}_{\mathrm{b}}$ and ASM values was 74 for physiological saline, 6 for $20 \mathrm{mM}, 12$ for $40 \mathrm{mM}, 6$ for $60 \mathrm{mM}, 14$ for $80 \mathrm{mM}, 6$ for $100 \mathrm{mM}$ and 16 for $120 \mathrm{mM}$ PEG. The ROIs used for the calculation of MK values were 57 for physiological saline, 6 for $20 \mathrm{mM}, 6$ for $40 \mathrm{mM}, 6$ for $60 \mathrm{mM}, 9$ for $80 \mathrm{mM}, 6$ for $100 \mathrm{mM}$ and 12 for $120 \mathrm{mM}$ PEG.

To evaluate the same values for the bio-phantom, the MK values were determined from three ROIs of $4 \times 1$ pixels in the cellular section inside the bio-phantom, and from ROIs of the same size in 6 areas of the physiological saline portion inside the phantom container.

With regard to $\mathrm{ADC}_{\mathrm{b}}$ and $\mathrm{ASM}$, the signal intensity was determined from four ROIs of $3 \times 3$ pixels selected in the cellular section of the bio-phantom. The signal intensity was also determined from $83 \times 3$-pixel ROIs located in the physiological saline portion of the phantom container. For DWI, signal intensity was determined from ROIs of $3 \times 3$ pixels in the images of RESOLVE-basic and RESOLVE-modify of each b-value. Each signal intensity value was logarithmically transformed. Then, the ADC value for each ROI was calculated from the inverse of the slope and the ASM value was calculated using Equation 3. The total ROIs used for the calculation of the ADC and ASM values was 12, 24 and 20 for cellularities of 46,522,778,
$158,267,558$ and $741,367,436$ cells/ml, respectively. The ROIs used for the calculation of MK values were 9, 18 and 15 for cellularities of 46,522,778, 158,267,558 and 741,367,436 cells/ml, respectively.

\section{Results}

The mean temperature and SD inside the bio-phantom was maintained at $37.2 \pm 0.7^{\circ} \mathrm{C}$ during imaging.

$A D C_{b}, M K$ and $A S M$ values of PEG phantoms. An increase in $\mathrm{PEG}$ concentration was associated with a decrease in $\mathrm{ADC}_{\mathrm{b}}$ values (Fig. 1A). Conversely, MK (Fig. 1B) and ASM (Fig. 1C) values increased in line with increasing PEG concentrations. The mean $\mathrm{ADC}_{\mathrm{b}}$ and $\mathrm{SD}$ values of the $0 \mathrm{mM}$ PEG phantom were $2.93 \pm 0.06 \times 10^{-3} \mathrm{~mm}^{2} / \mathrm{sec}$, and those for the $120 \mathrm{mM}$ PEG phantom were $0.64 \pm 0.01 \times 10^{-3} \mathrm{~mm}^{2} / \mathrm{sec}$. MK values corresponding to the $0 \mathrm{mM}$ and $120 \mathrm{mM}$ PEG phantom were $0.04 \pm 0.01$ and $1.20 \pm 0.14$, respectively. The ASM calculations for the $0 \mathrm{mM}$ and $120 \mathrm{mM}$ PEG concentrations yielded $1,412 \pm 965$ and $63,544 \pm 34,965\left(\mathrm{sec} / \mathrm{mm}^{2}\right)^{2}$, respectively.

$A D C_{b}, M K$ and $A S M$ values of bio-phantoms. The final cellularity of the bio-phantom ranged from $0.47 \times 10^{8}-7.41 \times 10^{8}$ cells $/ \mathrm{ml}$. The mean $\mathrm{ADC}_{\mathrm{b}}$ and $\mathrm{SD}$ values for the bio-phantoms were $2.31 \pm 0.05 \times 10^{-3} \mathrm{~mm}^{2} / \mathrm{sec}$ for the low-cellularity phantom and $0.79 \pm 0.05 \times 10^{-3} \mathrm{~mm}^{2} / \mathrm{sec}$ for the high-cellularity phantom (Fig. 2A). MK values ranged between $0.29 \pm 0.02$ and $1.27 \pm 0.03$ (Fig. 2B). ASM values ranged between $3,987 \pm 3,991$ and 48,039 $\pm 45,071\left(\mathrm{sec} / \mathrm{mm}^{2}\right)^{2}$ (Fig. 2C). The values for the bio-phantoms mostly lie within the range of the values 
A

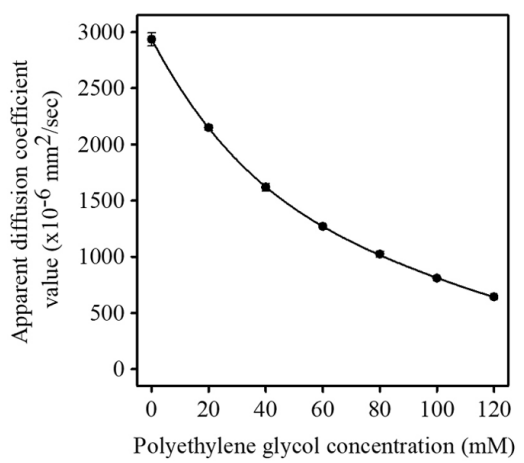

$\mathrm{B}$

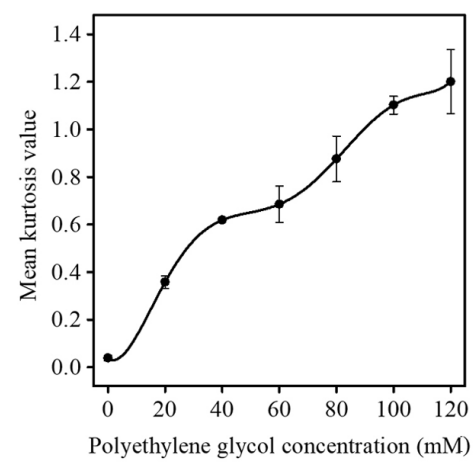

$\mathrm{C}$

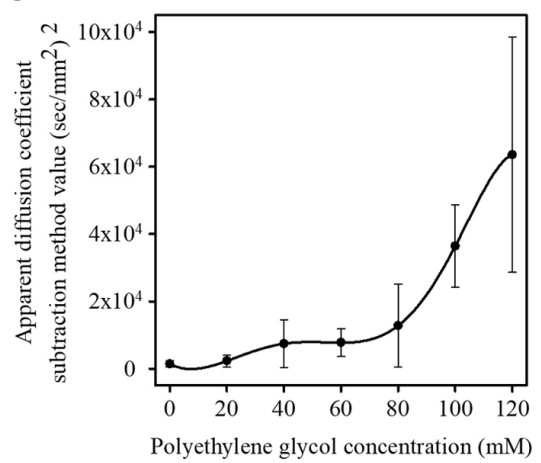

Figure 1. The association between PEG concentration and the values for $\mathrm{ADC}_{\mathrm{b}}$, MK and ASM. The horizontal axis indicates PEG concentration; the vertical axes indicate (A) $\mathrm{ADC}_{\mathrm{b}}$, (B) DKI and (C) ASM values. Data are presented as mean \pm standard deviation. The curves connecting the points indicate the regression curves for experimental data. PEG concentration is inversely proportional to $\mathrm{ADC}_{\mathrm{b}}$, and proportional to $\mathrm{DKI}$ and $\mathrm{ASM}$. PEG, polyethylene glycol; $\mathrm{ADC}$, apparent diffusion coefficient calculated for readout segmentation of long variable echo-trains-basic; MK, mean kurtosis; ASM, apparent diffusion coefficient subtraction method.

produced for the PEG phantoms. Fig. 2 indicates the correlation between cellularity and $\mathrm{ADC}_{\mathrm{b}}, \mathrm{MK}$, and $\mathrm{ASM}$ values; moreover, all data (except for the highest MK cellularity values) were ascertained to be within the range of the PEG phantom results.

Fig. 3 displays $\mathrm{ADC}_{\mathrm{b}}$, MK and ASM images of PEG and bio-phantoms. The $\mathrm{ADC}_{\mathrm{b}}$ and $\mathrm{ASM}$ images provide a higher resolution and less distortion compared with the MK image.

Empirical formula for calculating $A D C_{b}$ values of phantoms made using varying PEG concentrations. $\mathrm{ADC}_{\mathrm{b}}$ values for PEG phantoms with concentrations of $0,20,40,60,80,100$ a nd $120 \mathrm{mM}$ were plotted as in Fig. 1A. Using these data points, a regression curve and an empirical formula were constructed facilitating the calculation of the $\mathrm{ADC}_{\mathrm{b}}$ values from phantoms produced using varying PEG concentrations. The correlation factor $\left(\mathrm{R}^{2}\right)$ for this approximation was 1.00 . The empirical formula to calculate the $\mathrm{ADC}_{\mathrm{b}}$ value corresponding to any $\mathrm{PEG}$ concentration $(\mathrm{x})$ is as follows:

$$
\text { Formula 1: } \mathrm{ADC}_{\mathrm{b}}=\mathrm{a}_{1} \mathrm{x}^{4}+\mathrm{a}_{2} \mathrm{x}^{3}+\mathrm{a}_{3} \mathrm{x}^{2}+\mathrm{a}_{4} \mathrm{x}+\mathrm{a}_{5}
$$

$\left(a_{1}, 0.0000066786969390685 \times 10^{-6} ; a_{2},-0.0028279562\right.$ $3507326000 \times 10^{-6} ; \mathrm{a}_{3}, 0.484704389217086 \times 10^{-6} ; \mathrm{a}_{4}$, $\left.-48.0980342756939 \times 10^{-6} ; \mathrm{a}_{5}, 2,935.33497680997 \times 10^{-6}\right)$.

Empirical formula for calculating MK values of phantoms made using varying PEG concentrations. MK values for PEG phantoms with concentrations of $0,20,40,60,80,100$ and $120 \mathrm{mM}$ phantoms were plotted as in Fig. 1B. Using these data points, a regression curve and an empirical formula were created for calculating the MK values of phantoms made with varying PEG concentrations. The correlation factor $\left(\mathrm{R}^{2}\right)$ for this approximation was 1.00 . The empirical formula to calculate the MK value corresponding to any PEG concentration (x) is as follows:

Formula 2: $M K=b_{1} x^{6}+b_{2} x^{5}+b_{3} x^{4}+b_{4} x^{3}+b_{5} x^{2}+b_{6} x+b_{7}$

$\left(b_{1}, 0.00000000002574679427 ; b_{2},-0.000000009958\right.$ $61887106 ; b_{3}, 0.00000144033983524861 ; b_{4},-0.00009462031$ $704821070 ; b_{5}, 0.00263877966804671000 ; b_{6},-0.0089848099$ $\left.7102524000 ; b_{7}, 0.03857894408353960000\right)$.
Empirical formula for calculating ASM values of phantoms made using varying PEG concentrations. ASM values for PEG phantoms with concentrations of $0,20,40,60,80$, 100 and $120 \mathrm{mM}$ phantoms were plotted as in Fig. 1C. Using these data points, a regression curve and an empirical formula were created for calculating the ASM values of phantoms made with varying PEG concentrations. The correlation factor $\left(\mathrm{R}^{2}\right)$ for this approximation was 1.00. The empirical formula to calculate the ASM value corresponding to any PEG concentration $(\mathrm{x})$ is as follows:

\section{Formula 3: $\mathrm{ASM}=\mathrm{c}_{1} \mathrm{x}^{6}+\mathrm{c}_{2} \mathrm{x}^{5}+\mathrm{c}_{3} \mathrm{x}^{4}+\mathrm{c}_{4} \mathrm{x}^{3}+\mathrm{c}_{5} \mathrm{x}^{2}+\mathrm{c}_{6} \mathrm{x}+\mathrm{c}_{7}$}

$\left(\mathrm{c}_{1},-0.0000004118023456 ; \mathrm{c}_{2}, 0.0000866210497676 ;\right.$ $\mathrm{c}_{3},-0.0018022499380095 ; \mathrm{c}_{4},-0.5404589664540250 ; \mathrm{c}_{5}$, $34.3619915451854000 ; \mathrm{c}_{6},-423.5577461123460000 ; \mathrm{c}_{7}$, $1,411.8674192056000000)$.

Empirical formula for calculating $A D C_{b}$ values of biophantoms with any cellularity. $\mathrm{ADC}_{\mathrm{b}}$ values for bio-phantoms were plotted as in Fig. 2A. Using these data points, a regression curve and an empirical formula were created for calculating the $\mathrm{ADC}_{\mathrm{b}}$ values of bio-phantoms made with any cellularity. The correlation factor $\left(\mathrm{R}^{2}\right)$ for this approximation was 1.00. The empirical formula to calculate the $\mathrm{ADC}_{\mathrm{b}}$ value corresponding to any cellularity (y) is as follows:

$$
\text { Formula 4: } \mathrm{ADC}_{\mathrm{b}}=\mathrm{d}_{1} \ln (\mathrm{y})+\mathrm{d}_{2}
$$

$\left(\mathrm{d}_{1},-552.230079387058 \times 10^{-6} ; \mathrm{d}_{2}, 12,081.0508279336\right.$ $\left.00 \times 10^{-6}\right)$.

Empirical formula for calculating MK values of bio-phantoms with varying cellularity. MK values for bio-phantoms are plotted in Fig. 2B and the data points were used to create a regression curve and an empirical formula for the calculation of MK values of bio-phantoms made with varying cellularity. The correlation factor $\left(R^{2}\right)$ for this approximation was 1.00 . The empirical formula to calculate the MK value corresponding to varying cellularity (y) is as follows: 
A

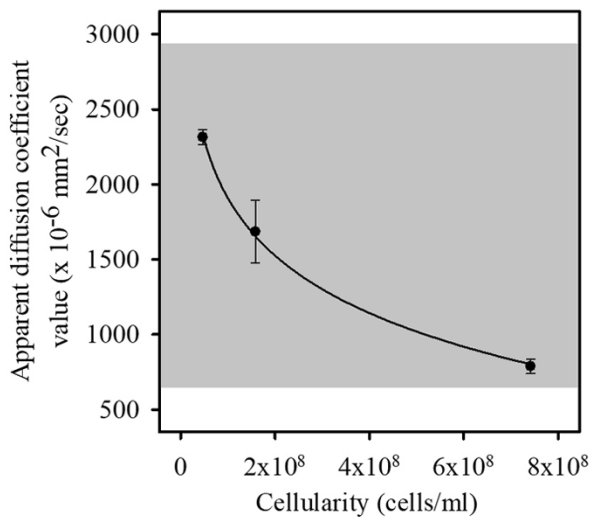

B

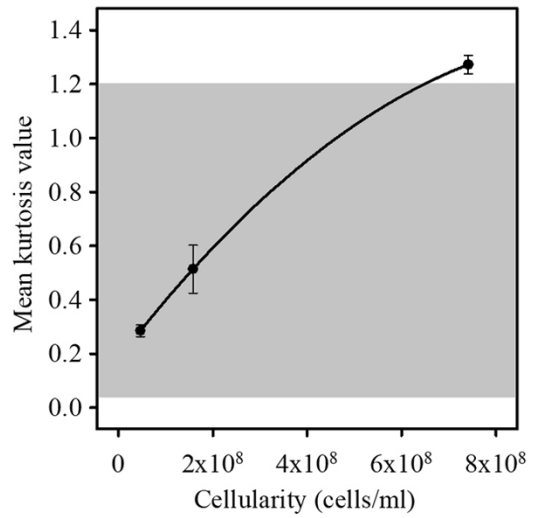

$\mathrm{C}$

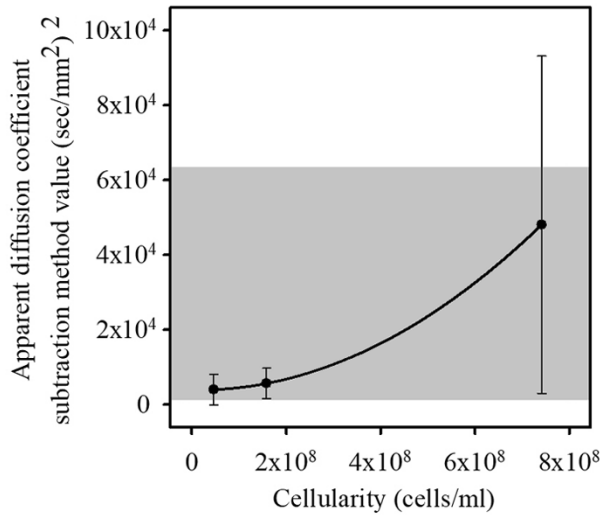

Figure 2. The association between cellularity and the values for $\mathrm{ADC}_{\mathrm{b}}, \mathrm{MK}$ and $\mathrm{ASM}$. Horizontal axes indicates cellularity and vertical axes indicate (A) $\mathrm{ADC}_{\mathrm{b}}$, (B) MK and (C) ASM values. Data are presented as mean \pm standard deviation. Curves connecting the points indicate the regression curves for the experimental data. The shaded area in each graph represents the range of $\mathrm{ADC}_{\mathrm{b}}$, MK and ASM values covered by the PEG phantom described in this paper. $\mathrm{ADC}_{\mathrm{b}}$, apparent diffusion coefficient calculated for readout segmentation of long variable echo-trains-basic; MK, mean kurtosis; ASM, apparent diffusion coefficient subtraction method; PEG, polyethylene glycol.
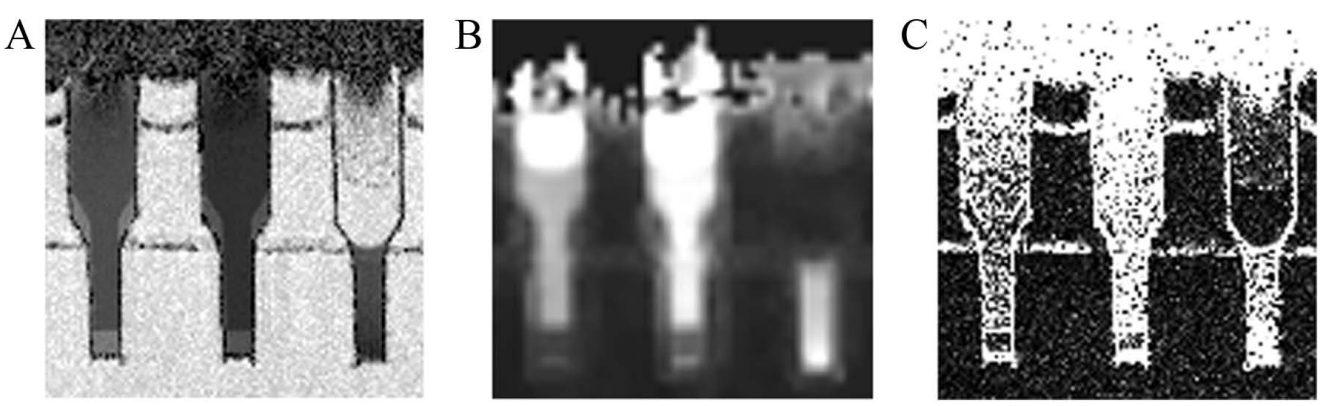

Figure 3. $\mathrm{ADC}_{\mathrm{b}}$, MK and ASM images of PEG and bio-phantoms. (A) $\mathrm{ADC}_{\mathrm{b}}$ image. (B) MK image. (C) ASM image. In each figure, the left, middle and right phantoms are $80 \mathrm{mM}$ PEG, $120 \mathrm{mM}$ PEG and high-cellularity (741,367,436 cells/ml) bio-phantom, respectively. $\mathrm{ADC}_{\mathrm{b}}$ and $\mathrm{ASM}$ images provide a higher resolution and less distortion compared to the MK image, due to the use of RESOLVE sequence. $\mathrm{ADC}_{\mathrm{b}}$, apparent diffusion coefficient calculated for readout segmentation of long variable echo-trains-basic; MK, mean kurtosis; ASM, apparent diffusion coefficient subtraction method; PEG, polyethylene glycol; RESOLVE, readout segmentation of long variable echo-trains.

Formula 5: $\mathrm{MK}=\mathrm{e}_{1} \mathrm{y}^{2}+\mathrm{e}_{2} \mathrm{y}+\mathrm{e}_{3}$

$\left(\mathrm{e}_{1},-0.00000000000000000108 ; \mathrm{e}_{2}, 0.00000000226921884\right.$ $\left.624 ; \mathrm{e}_{3}, 0.18153679067533800000\right)$.

Empirical formula for calculating ASM values of bio-phantoms with varying cellularity. The ASM values for bio-phantoms are exhibited in Fig. 2C. Using these data points, a regression curve and an empirical formula were created to calculate the ASM values of bio-phantoms made with varying cellularity. The correlation factor $\left(\mathrm{R}^{2}\right)$ for this approximation was 1.00 . The empirical formula to calculate the ASM value corresponding to varying cellularity $(\mathrm{y})$ is as follows:

$$
\text { Formula 6: } \mathrm{ASM}=\mathrm{f}_{1} \mathrm{y}^{2}+\mathrm{f}_{2} \mathrm{y}+\mathrm{f}_{3}
$$

$\left(f_{1}, 0.00000000000008320968 ; f_{2},-0.0000021617730\right.$ 7991540; $\left.\mathrm{f}_{3}, 3,907.60230188793000000000\right)$.

\section{Discussion}

In the present study, it was revealed that polyethylene glycol (PEG) phantoms are able to mimic a wide range of values, not only for the apparent diffusion coefficient (ADC), but also for restricted diffusion. Formulae were constructed in order to calculate PEG concentrations corresponding to arbitrary ADC, mean kurtosis (MK) and ADC subtraction method (ASM) values. The current results indicate that PEG phantoms may be used as a standard phantom for restricted diffusion with the desired MK and ASM values in MRI.

PEG, which is used as the base material for our standard phantom for ADC, MK and ASM, is a safe material and used as a base in many everyday products, including lapactics, skin creams and cosmetic emulsifiers (4). PEG phantom is safer than previously reported ADC standard phantoms-gelatinous substances such as agar (10), agarose (11) and polyacrylamide (12); and liquid solution materials such as ethanol (13), acetone (14-17), Gd-DTPA solution (18) and cupric sulfate solution (19). PEG phantom is also less expensive and easier to make than the previously reported ADC standard phantoms.

Matsumoto et al used a bio-phantom using tumor cells to visualize restricted diffusion (9). Bio-phantoms are complicated to create, time consuming and expensive, and impossible to use for a long time. The PEG phantom is ready to make, cheap and safe and can be stored for a long time. 
PEG is a high molecular compound with a structure polymerized by ethylene glycol. Sehy et al (20) revealed that injecting PEG into cells decreased ADC, suggesting its suppressive action on water diffusion. In a previous study, Matsuya et al (4) proposed the use of PEG phantoms as the standard phantom for apparent diffusion coefficient (ADC). Empirical formulae were then constructed to calculate the PEG concentration at any measured temperature to obtain arbitrary ADC values for 1.5T MRI. Following the formulae, any phantoms with arbitrary ADC values can be made to mimic any tumors at any temperature.

ADC represents free and restricted diffusion in MR images. In recent years, new MR imaging techniques representing restricted diffusion have been developed. Of these, DKI is often considered the most promising and has been suggested as an alternative to ADC maps in clinical trials (7). A typical DKI protocol consists of a total of 60-70 images; and more data-demanding protocols are also available. Post-DKI data processing also requires significant time, especially in patients requiring urgent attention (e.g., critical cases and children). However, the superiority of DKI imaging supports developing the clinical and preclinical studies for DKI. Recently, faster imaging techniques for DKI have been already developed (21).

In a recent study, ASM was proposed as a new technique for restricted diffusion (8). ASM enables the observation of water-molecule diffusion with a space-separation resolution of $1 \mu \mathrm{m}$. ASM also produces a high image quality (without distortion) using readout segmentation of long variable echo-train (RESOLVE) sequences (22). Under the imaging conditions of this study, the resolution of ASM was higher than that of DKI (Fig. 3). If ASM's image quality were set to the same as that of DKI, ASM would take a much shorter imaging time than DKI in this study. The PEG phantom created in the current study is the first reported standard restricted diffusion phantom for DKI and ASM.

The MK values for the bio-phantoms predominantly fall within the range of the values for the PEG phantoms. Although the phantoms in the current study did not cover the upper limit for the bio-phantoms' MK (1.27 \pm 0.03$)$, the maximum cell density covered by our phantom was calculated using empirical formula 5 to be $650,892,050$ cells $/ \mathrm{ml}$.

MK values in clinical studies using 3T MRI have been previously reported for various tumors; for example, $\mathrm{MK}$ for grade II glioma is $0.50 \pm 0.08$ (23); for rectal cancer $1.000 \pm 0.112$ (24); for squamous cell carcinoma $0.917 \pm 0.144$; and for olfactory neuroblastoma 1.209 \pm 0.262 (25). ADC values for common tumors in clinical studies using 3T MRI vary between $0.62 \times 10^{-3} \mathrm{~mm}^{2} / \mathrm{sec}$ (brain lymphoma) and $2.60 \times 10^{-3} \mathrm{~mm}^{2} / \mathrm{sec}$ (neoplastic cystic lesions in the pancreas) (26). The current phantom covers the majority of these values and can therefore be used to mimic most common tumor types.

The ASM values of the bio-phantoms fall completely within the range of the values for the PEG phantoms, suggesting the usability of the phantom in the present study for future ASM experiments. To the best of our knowledge, this is the first paper to present a phantom covering ASM values of biological tissue since the advent of ASM.

There are a few limitations to this study. The equations in the present study were constructed according to values acquired using 3T MRI and sequences available for this experiment.
Further examination may be necessary to clarify the effect of imaging conditions, such as the type of MRI devices and sequences used, to ensure the reproducibility of the current results. Furthermore, the mechanism by which PEG mimics restricted diffusion is yet to be elucidated, although there is a possibility that PEG molecules exert a limiting effect on the movement of water molecules. The PEG phantom, however, may have limitations in mimicking restricted diffusion beyond a certain point, since it does not have a concrete restricting structure (such as a membrane), but it was revealed to mimic restricted diffusion within a certain range of clinically reported DKI values. Therefore, this technique may prove useful for future experiments as long as the truncated range is accurately accounted for.

Using the newly developed MRI phantom, instead of imaging the actual tumor in the patient's body, a new method for imaging restricted diffusion might be developed by imaging the phantom with the MK value and ADC value of the tumor. Unlike clinical trials with patients, this phantom contributes to safe and cost-effective research. Improvement of imaging methods for future clinical dissemination of restricted diffusion imaging is expected worldwide and we believe this phantom will contribute to such research.

In conclusion, it was revealed that PEG decreased restricted diffusion and this indicates the potential of the PEG phantom as a standard phantom for restricted diffusion MRIs.

\section{Acknowledgements}

Not applicable.

\section{Funding}

The present study was partially supported by Grants-in-Aid for Scientific Research (grant nos. C22591335, 15K09924, and 19K0809801) from the Ministry of Health, Labour and Welfare of Japan.

\section{Availability of data and materials}

The materials and datasets used and/or analyzed during the current study are available from the corresponding author on reasonable request.

\section{Authors' contributions}

AKh, YY and MK conceived and designed the study, processed the data and wrote the article. AKh, MK, YY, IS, $\mathrm{BOB}, \mathrm{KH}, \mathrm{MB}, \mathrm{NT}, \mathrm{KK}, \mathrm{KS}, \mathrm{HI}, \mathrm{AKu}, \mathrm{TM}, \mathrm{SO}, \mathrm{SK}$ and JA participated in conducting the experiments. IS, AKh, KH, MB and NT edited the article. All authors read and approved the final version of the manuscript.

\section{Ethics approval and consent to participate}

Not applicable.

\section{Patient consent for publication}

Not applicable. 


\section{Competing interests}

The authors declare that there are no competing interests regarding the publication of the present manuscript.

\section{References}

1. Drake-Pérez M, Boto J, Fitsiori A, Lovblad K and Vargas MI: Clinical applications of diffusion weighted imaging in neuroradiology. Insights Imaging 9: 535-547, 2018.

2. Stecco A, Buemi F, Iannessi A, Carriero A and Gallamini A: Current concepts in tumor imaging with whole-body MRI with diffusion imaging (WB-MRI-DWI) in multiple myeloma and lymphoma. Leuk Lymphoma 59: 2546-2556, 2018.

3. Tang L and Zhou XJ: Diffusion MRI of cancer: From low to high b-values. J Magn Reson Imaging 49: 23-40, 2019.

4. Matsuya R, Kuroda M, Matsumoto Y, Kato H, Matsuzaki H, Asaumi J, Murakami J, Katashima K, Ashida M, Sasaki T, et al A new phantom using polyethylene glycol as an apparent diffusion coefficient standard for MR imaging. Int J Oncol 35: 893-900, 2009

5. Le Bihan D and Iima M: Diffusion magnetic resonance imaging: What water tells Us about biological tissues. PLoS Biol 13 . e1002203, 2015.

6. Le Bihan D and Johansen-Berg H: Diffusion MRI at 25: Exploring brain tissue structure and function. Neuroimage 61 : 324-341, 2012.

7. Jensen JH and Helpern JA: MRI quantification of non-Gaussian water diffusion by kurtosis analysis. NMR Biomed 23: 698-710, 2010.

8. Yoshimura Y, Kuroda M, Sugianto I, Khasawneh A, Bamgbose BO, Hamada K, Barham M, Tekiki N, Kurozumi A, Matsushita T, et al: Development of a novel method for visualizing restricted diffusion using subtraction of apparent diffusion coefficient values. Mol Med Rep 20: 2963-2969, 2019.

9. Matsumoto Y, Kuroda M, Matsuya R, Kato H, Shibuya K, Oita M, Kawabe A, Matsuzaki H, Asaumi J, Murakami J, et al: In vitro experimental study of the relationship between the apparent diffusion coefficient and changes in cellularity and cell morphology. Oncol Rep 22: 641-648, 2009.

10. Li TQ, Kim DH and Moseley ME: High-resolution diffusion-weighted imaging with interleaved variable-density spiral acquisitions. J Magn Reson Imaging 21: 468-475, 2005.

11. Ogura A, Maeda F, Miyai A, Hayashi K and Hongoh T: Effect of vibration caused by time-varying magnetic fields on diffusion-weighted MRI. Nihon Hoshasen Gijutsu Gakkai Zasshi 62: 565-569, 2006 (In Japanese).

12. Hirsch JG, Bock M, Essig M and Schad LR: Comparison of diffusion anisotropy measurements in combination with the flair-technique. Magn Reson Imaging 17: 705-716, 1999.

13. Deng J, Omary RA and Larson AC: Multishot diffusion-weighted Splice Propeller MRI of the abdomen. Magn Reson Med 59: 947-953, 2008.
14. Yoshikawa T, Kawamitsu H, Mitchell DG, Ohno Y, Ku Y, Seo Y, Fujii M and Sugimura K: ADC measurement of abdominal organs and lesions using parallel imaging technique. Am J Roentgenol 187: 1521-1530, 2006.

15. Bammer R, Stollberger R, Augustin M, Simbrunner J, Offenbacher H, Kooijman H, Ropele S, Kapeller P, Wach P, Ebner F and Fazekas F: Diffusion-weighted imaging with navigated interleaved echo-planar imaging and a conventional gradient system. Radiology 211: 799-806, 1999.

16. Brockstedt S, Thomsen C, Wirestam R, Holtas S and Stahlberg F: Quantitative diffusion coefficient maps using fast spin-echo MRI. Magn Reson Imaging 16: 877-886, 1998.

17. Lebihan D, Breton E, Lallemand D, Aubin ML, Vignaud J and Lavaljeantet M: Separation of diffusion and perfusion in intravoxel incoherent motion MR imaging. Radiology 168 : 497-505, 1988.

18. Moteki T and Ishizaka H: Evaluation of cystic ovarian lesions using apparent diffusion coefficient calculated from turboFLASH MR images. Br J Radiol 71: 612-620, 1998.

19. Kinoshita Y, Iriguchi N and Yokota A: Study of diffusion phenomenon using an experimental magnetic resonance system (SIS 200/400) for small animals-reliability and apparent diffusion coefficient of normal animals. J UOEH 17: 261-269, 1995.

20. Sehy JV, Ackerman JJH and Neil JJ: Apparent diffusion of water, ions, and small molecules in the Xenopus oocyte is consistent with Brownian displacement. Magn Reson Med 48: 42-51, 2002.

21. Hansen B and Jespersen SN: Recent developments in fast kurtosis imaging. Front Phys 5: 40, 2017.

22. Yoshimura Y, Kuroda M, Sugiantoc I, Bamgbosec BO, Miyahara K, Ohmura Y, Kurozumi A, Matsushita T, Ohno S, Kanazawa $S$ and Asaumi J: The usefulness of readout-segmented echo-planar imaging (RESOLVE) for bio-phantom imaging using 3-tesla clinical MRI. Acta Med Okayama 72: 53-59, 2018.

23. Delgado A, Fahlström M, Nilsson M, Berntsson SG, Zetterling M, Libard S, Alafuzoff I, Van Westen D, Lätt J, Smits A and Larsson EM: Diffusion kurtosis imaging of gliomas grades II and III-a study of perilesional tumor infiltration, tumor grades and subtypes at clinical presentation. Radiol Oncol 51: 121-129, 2017.

24. Sun Y, Xiao Q, Hu F, Fu C, Jia H, Yan X, Xin C, Cai S, Peng W, Wang X, et al: Diffusion kurtosis imaging in the characterisation of rectal cancer: Utilizing the most repeatable region-of-interest strategy for diffusion parameters on a 3T scanner. Eur Radiol 28: 5211-5220, 2018

25. Xiao Z, Tang Z, Qiang J, Qian W, Zhong Y, Wang R, Wang J, Wu L and Tang W: Differentiation of olfactory neuroblastomas from nasal squamous cell carcinomas using MR diffusion kurtosis imaging and dynamic contrast-enhanced MRI. J Magn Reson Imaging 47: 354-361, 2018.

26. Hara M, Kuroda M, Ohmura Y, Matsuzaki H, Kobayashi T, Murakami J, Katashima K, Ashida M, Ohno S and Asaumi JI: A new phantom and empirical formula for apparent diffusion coefficient measurement by a 3 tesla magnetic resonance imaging scanner. Oncol Lett 8: 819-824, 2014. 